\title{
Trial Summary Parameter Name of the Reference Terminology
}

National Cancer Institute

\section{Source}

National Cancer Institute. Trial Summary Parameter Name of the Reference

Terminology. NCl Thesaurus. Code C117460.

The textual description of the terminology system that is being used for the trial summary parameter. 